\title{
Pharmacists' Bleed Risk Tool and Treatment Preferences Prior to Initiating Anticoagulation in Patients With Nonvalvular Atrial Fibrillation: A Cross-Sectional Survey
}

Devada Singh-Franco, PharmD, CDE, Genevieve Hale, PharmD, BCPS, BCCP, CPh, Tina Joseph, PharmD, BCACP, and William R. Wolowich, PharmD

\section{ABSTRACT}

Objective: To determine pharmacists' preferences in bleed risk tool (BRT) usage and gastroprotection when bleed risk was lower than or equal to stroke risk in patients with nonvalvular atrial fibrillation and who were candidates for oral anticoagulation therapy (warfarin or direct oral anticoagulants [DOACs]).

Methods: A survey consisting of 4 domains (demographics, clinical experience, BRT usage, and treatment preferences based on cases where bleed risk was lower than or equal to stroke risk) was developed. The anonymous survey was disseminated via REDCap software to members of the American College of Clinical Pharmacy ambulatory care and cardiology Practice-based Research Networks. Descriptive statistics were calculated for all study variables and inferential statistics were employed as necessary.
M anagement of patients with nonvalvular atrial fibrillation (NVAF) with oral anticoagulation therapy (OACT) requires constant attention to maintain a balance between preventing strokes and minimizing bleeds. Several validated bleed risk tools (BRTs) available for use in NVAF patients include HAS-BLED,

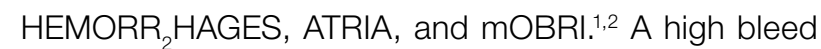
risk score is not a contraindication to OACT, but, prior to and throughout therapy, bleed risk should be assessed and modifiable risk factors addressed. ${ }^{3}$ While intraluminal gastrointestinal (GI) bleeds are not considered a critical bleed site, they are a common complication of chronic OACT and can result in hemodynamic compromise and permanent discontinuation of therapy., ${ }^{4,5} 3233$
Results: Of 165 BRT users, 97\% preferred HAS-BLED. When bleed risk was lower than stroke risk, 151 respondents chose either DOACs (65\%) or warfarin (35\%); $15 \%$ added gastroprotection. When bleed risk was equal to stroke risk, 141 respondents chose DOACs (50\%), warfarin (45\%), or aspirin (5\%); $40 \%$ added gastroprotection.

Conclusion: In addition to BRT usage, pharmacists were judicious in their recommendation to add gastroprotection and would consider doing so if there was a specific indication. As more than $80 \%$ of extracranial bleeds are gastrointestinal bleeds and most BRTs are nonspecific for predicting these bleeds, randomized, prospective studies stratified by HAS-BLED and stroke risk scores are needed to provide further guidance on the efficacy and safety of oral anticoagulation therapy with or without gastroprotection.

Keywords: NVAF; gastroprotection; proton pump inhibitors; warfarin; oral anticoagulants.

patients with nonvariceal upper Gl bleeds (2005-2016), the adjusted odds ratio of hospital admission, transfusion, and re-bleeding while on OACT (warfarin, heparin, or apixaban) was 3.48, 2.53, and 2.26, respectively. ${ }^{6}$ Addition of acid-suppressive therapy with a proton pump inhibitor (PPI) or histamine-2 receptor antagonist $\left(\mathrm{H}_{2} \mathrm{RA}\right)$ in NVAF patients at increased risk for upper Gl bleeds and receiving OACT may result in fewer bleeds.,8

Pharmacists play an integral part in managing patients on warfarin,,-11 and data on their role in managing patients receiving direct oral anticoagulants (DOACs) are

From Nova Southeastern University College of Pharmacy, Fort Lauderdale, FL. 
increasing. ${ }^{12-16}$ Inpatient pharmacists actively participate in multidisciplinary collaborative teams and use clinical decision-support systems or enhanced monitoring to ensure safe prescribing of high-risk medications. ${ }^{12,15,16}$ Pharmacist-managed, outpatient-based anticoagulation services in patients on warfarin were associated with lower rates of bleeding and thromboembolic events and lower health care utilization versus routine care. ${ }^{17}$ However, it is unclear how pharmacists manage patients who are candidates for OACT but who may be at increased risk for upper Gl bleeds. Using a US-based survey, the investigators sought to determine pharmacists' preferences in BRT usage and gastroprotection when bleed risk was lower than or equal to stroke risk.

\section{Methods}

This cross-sectional study was conducted after receiving approval by Nova Southeastern University's Institutional Review Board. The survey consisted of 16 items divided into 4 domains: demographics, clinical experience, use of BRTs, and treatment preferences based on cases where bleed risk was lower than or equal to stroke risk (Figure 1). Queries were multiple choice and allowed for free-text input when "Other" was selected. Licensed pharmacists $\geq 18$ years of age who routinely provided care to patients with NVAF were eligible to participate in the study. Participants who reported using a BRT (users) completed all study domains, while participants who reported not using a BRT (nonusers) completed domains 1 through 3 only.

An invitation containing the survey link was sent to the American College of Clinical Pharmacy ambulatory care ( $n=2237)$ and cardiology $(n=1318)$ pharmacists listed in the organization's Practice-based Research Networks. The survey was administered in the United States between April and June 2016 via Research Electronic Data Capture (REDCap) software, a secure Web application for building and managing online surveys designed to support data collection for research studies. ${ }^{18}$

Survey responses were downloaded, and data were analyzed using NCSS 2019 Statistical Software, LLC (Kaysville, UT). Descriptive statistics were calculated for all study variables. Demographic and clinical experience data for the group that used a BRT versus the group that did not were compared using Pearson's chi- square, ANOVA, or the Cochran-Armitage test for trends. Logistic regression with hierarchical forward selection with switching was used to identify predictors of drug selection and use of gastroprotection.

\section{Results}

Of 230 respondents who completed the survey (response rate $6.5 \%), 165$ (72\%) used a BRT and 65 (28\%) did not. No significant differences were found for age, gender, duration in clinical practice, the percentage of time spent in patient care, or practice specialty between users and nonusers (Table). The median age of users was 32 years; $68 \%$ were females; the median duration in clinical practice was 6 years; $75 \%$ of their time was spent in clinical practice; and clinical settings included ambulatory care, cardiology, and internal medicine. A significant difference was found for practice region between users versus nonusers $(P=0.014)$. Respondents who managed more than 200 NVAF patients per year used a BRT more often than those who managed fewer than 100 NVAF patients per year $(P=0.001)$.

Of those who used a BRT, $97 \%$ utilized the HAS-BLED tool $(n=160)$. The remainder used HEMORR ${ }_{2}$ HAGES $(n=3)$, ATRIA $(n=1)$, and mOBRI $(n=1)$. Reasons for choosing HAS-BLED included "familiarity/ease-of-use," "preference by institution/clinical team," and the fact that it was a "validated tool for NVAF."

When bleed risk was lower than stroke risk, 151 of 165 users (92\%) chose a treatment option (Figure 2). Of those, 65\% chose a DOAC and 35\% chose warfarin. Fourteen respondents chose "other" and explained that they "would initiate OACT after weighing patient factors and preferences." When a DOAC was selected, 9\% $(n=9)$ chose PPI co-therapy and $4 \%(n=4)$ chose a $\mathrm{H}_{2} \mathrm{RA}$. When warfarin was selected, $13 \%(n=7)$ chose PPI co-therapy and $4 \%(n=2)$ chose $a H_{2} R A$. Respondents who chose gastroprotection did not provide reasons for doing so, but those who did not add it explained that they "would add gastroprotection only if patient is also on an NSAID or has a history of Gl bleed" or cited "patient preference." Specific to warfarin, some respondents would not add gastroprotection, as anticoagulation with warfarin is "easily reversed."

When bleed risk was equal to stroke risk, 141 of 165 users (85\%) chose a treatment option (Figure 3). Fifty 
Figure 1. Survey tool. DOAC, direct-acting oral anticoagulant; NVAF, nonvalvular atrial fibrillation.

\section{Demographics}

1. Please provide your age in years (drop-down menu)

2. Please check your gender
a. Male
b. Female

3. In which state do you currently practice? (drop-down menu)

4. Which of the following best describe your specialty?
a. Cardiology Pharmacist
b. Ambulatory Care Pharmacist
c. Internal Medicine Pharmacist
d. Other

\section{Clinical Experience}

5. How many years have you been in clinical practice? (drop-down menu)

6. About what percent of your time is spent in clinical practice or direct patient care as opposed to non-direct patient care activities, including order verification?

a. \% of Time

\section{Bleed Risk Tool Usage}

7. Do you currently use a risk scoring tool to determine the risk of bleeding in NVAF patients?
a. Yes
b. No (did not view "cases")

8. Which scoring tool do you currently use to determine the risk of bleeding in NVAF patients?
a. HAS-BLED
b. HEMORR 2 HAGES
c. ATRIA
d. Other

\section{"Cases"}

9. If NVAF patient is a candidate for anticoagulation, and bleed risk score is less than stroke risk score, which of the following treatment options would you recommend?
a. No treatment
b. Warfarin
c. DOAC
d. Aspirin
e. Aspirin $+\mathrm{P} 2 \mathrm{Y}_{12}$ inhibitor
f. Other

9a. With or without gastroprotection?

9b. If with gastroprotection, select
a. Proton pump inhibitors
b. Histamine-2 receptor antagonists
c. Other

10. If NVAF patient is a candidate for anticoagulation, and bleed risk score is equal to stroke risk score, which of the following treatment options would you recommend?
a. No treatment
b. Warfarin
c. DOAC
d. Aspirin
e. Aspirin $+\mathrm{P}^{2} \mathrm{Y}_{12}$ inhibitor
f. Other

10a. With or without gastroprotection?

10b. If with gastroprotection, select
a. Proton pump inhibitors
b. Histamine-2 receptor antagonists
c. Other

percent chose DOACs, 45\% chose warfarin, and 5\% chose aspirin. Logistic regression analysis (outcome DOAC versus warfarin area-under-ROC curve, 0.67) showed that as the number of NVAF patients seen in 12 months increased, respondents were more likely to select a DOAC over warfarin (odds ratio, 1.7; 95\% Cl, 1.1-2.5). Therefore, for every 50-patient increase per year, the probability of recommending a DOAC increased 1.7-fold.

Of respondents who selected either a DOAC or warfarin, $38 \%(n=50)$ also added gastroprotection (Figure 3). When a DOAC was selected, 34\% ( $n=24)$ favored PPI co-therapy and $7 \%(n=5)$ chose a $\mathrm{H}_{2} R A$. When warfarin was selected, 19\% ( $n=12$ ) favored PPI co-therapy, while 13\% ( $n=8$ ) chose a $\mathrm{H}_{2} \mathrm{RA}$. Rationale for choosing gastropro- tection, regardless of OACT selection, included "stroke is more devastating, so if patient wants to continue treatment, but knew risks of bleeding were similar, would recommend gastroprotection to help minimize bleeding risk" and "patient-specific consideration." Rationales for not choosing gastroprotection included "would add gastroprotection only if patient is on dual antiplatelet therapy or has another indication"; "in most patients, stroke risk outweighs bleed risk so no need for gastroprotection unless there is a stated reason"; "would use apixaban as has lowest bleeding rate of all DOACs in clinical trials"; and "gastroprotection has not been shown to be beneficial in large scale trials."

Eight respondents chose aspirin because it was "easy and relatively low cost." Twenty-four respondents chose 
Table. Respondent Demographics

\begin{tabular}{|c|c|c|c|}
\hline & $\begin{array}{l}\text { Users } \\
(n=165)\end{array}$ & $\begin{array}{c}\text { Nonusers } \\
(n=65)\end{array}$ & $P$ Value \\
\hline Age, median (IQR), y & $32(28-38)$ & $33(30-38.5)$ & $0.4^{\mathrm{a}}$ \\
\hline \multicolumn{4}{|l|}{ Gender } \\
\hline Female, no. (\%) & $113(68)$ & $41(63)$ & \multirow[t]{2}{*}{$0.4^{b}$} \\
\hline Male, no. (\%) & $52(32)$ & $24(37)$ & \\
\hline \multicolumn{4}{|l|}{ Practice region, no. (\%) } \\
\hline Midwest & $66(40)$ & $17(26)$ & \multirow{5}{*}{$0.014^{b}$} \\
\hline Southeast & $49(30)$ & $21(32)$ & \\
\hline Northeast & $20(12)$ & $19(29)$ & \\
\hline West & $21(13)$ & $4(6)$ & \\
\hline Southwest & $9(5)$ & $4(6)$ & \\
\hline \multicolumn{4}{|l|}{ Specialties, no. (\%) } \\
\hline Ambulatory Care & $63(38)$ & $24(37)$ & \multirow{4}{*}{$0.1^{b}$} \\
\hline Cardiology & $52(31)$ & $12(19)$ & \\
\hline Internal Medicine & $34(21)$ & $19(29)$ & \\
\hline Other & $16(10)$ & $10(15)$ & \\
\hline Duration in clinical practice, median (IQR), y & $6(3-11)$ & $6(3-15)$ & $0.4^{\mathrm{a}}$ \\
\hline Percent time spent in direct patient care, median (IQR) & $75(50-90)$ & $70(50-82.5)$ & $0.3^{\mathrm{a}}$ \\
\hline \multicolumn{4}{|l|}{ NVAF patients seen in a 12-month period, no. (\%) } \\
\hline Less than 100 & $48(29)$ & $33(51)$ & \multirow{3}{*}{$0.001^{c}$} \\
\hline $100-199$ & $56(34)$ & $18(28)$ & \\
\hline 200 or more & $61(37)$ & $14(21)$ & \\
\hline \multicolumn{4}{|l|}{ IQR, interquartile range; NVAF, nonvalvular atrial fibrillation. } \\
\hline \multicolumn{4}{|l|}{ 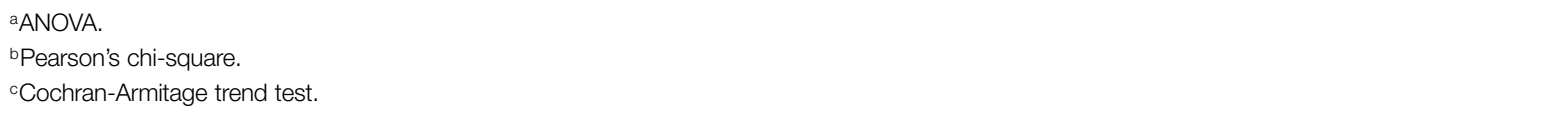 } \\
\hline
\end{tabular}

"other" and explained that the choice of OACT depended on patient preference after they had discussed stroke and bleed risk with the patient and/or determined the etiology driving bleed risk.

\section{Discussion}

This is the first national survey exploring US pharmacists' preferences in BRT usage and treatment based on bleed risk. Pharmacists preferred the HAS-BLED tool and considered patient-specific factors and evidence-based data when weighing the risk-benefit of OACT with or without gastroprotective therapy.

Similar to our findings, where three-quarters of pharmacists used a BRT, a recent Medscape/American College of Cardiology (ACC) survey reported that $74 \%$ of cardiologists used a BRT (eg, HAS-BLED) always/most of the time or sometimes to assess a patient's overall risk of bleeding prior to initiating DOAC therapy; $27 \%$ never or rarely used a bleed risk score before prescribing DOACs. ${ }^{19}$ Although reasons for BRT preference were not provided, they may be similar to those reported by our respondents (ie, familiarity/ease-of-use). In both surveys, rationales for not using a BRT were not obtained, but possible reasons include lack of confidence with bleed risk calculators, ${ }^{20}$ inconsistent implementation of comprehensive assessments (stroke risk, bleed risk, and medication-related issues prior to decision-making), ${ }^{21}$ and nonspecific guideline recommendations. ${ }^{22}$

More recently, a network meta-analysis found that HAS-

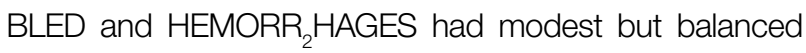
sensitivity (defined as the ratio between the number of major bleeding events in high-risk stratification and the total 


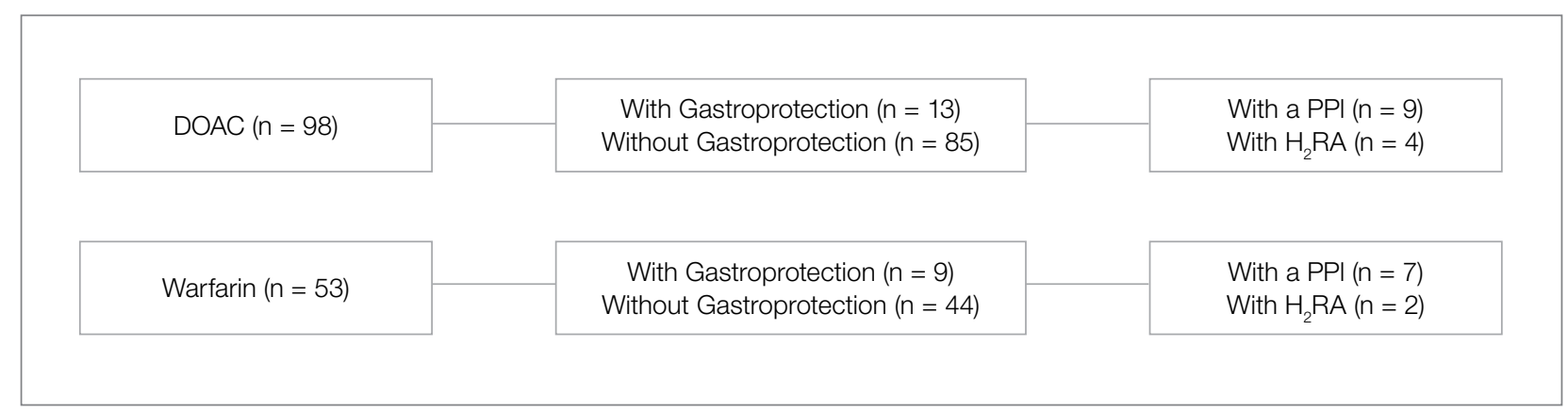

Figure 2. Pharmacists' treatment preferences if bleed risk is less than stroke risk $(n=151)$. Data of 14 pharmacists are not included in figure, as they chose "other" treatment.

DOAC, direct-acting oral anticoagulant (apixaban [ $\mathrm{n}=66]$; rivaroxaban [ $=25]$; other $[\mathrm{n}=7]$ ); PPI, proton pump inhibitor; $\mathrm{H}_{2} \mathrm{RA}$, histamine-2 receptor antagonist.

number of bleeding events) and specificity (defined as the ratio between the number of nonmajor bleeding events in the low-risk population and total nonbleeding events) for predicting major bleeding events. ${ }^{2,3}$ Several respondents did comment that, although HAS-BLED was imprecise and only studied with warfarin, it was necessary to identify bleed risk in a patient starting a high-risk medication, and that the ACC anticoagulation application uses HAS-BLED with $\mathrm{CHA}_{2} \mathrm{DS}_{2} \mathrm{VASc}$ along with clinical trial data to estimate stroke risk and bleed risk, with projected risk reduction (strokes) and risk increases (bleeds) expected with each treatment (www.acc.org/tools-and-practice-support/ mobile-resources/features/anticoag-evaluator). The 2019 AHA/ACC/HRS atrial fibrillation guideline recommends that HAS-BLED scores be used to assess bleed risk in patients for whom anticoagulation is being considered, and that the need for and choice of OACT should be periodically reevaluated to reassess stroke and bleed risks. ${ }^{23}$

Although more than $80 \%$ of extracranial bleeds are Gl bleeds, ${ }^{24}$ most BRTs are nonspecific for predicting Gl bleeds. Indeed, one respondent used a spreadsheet with several BRTs to maximize treatment guidance for patients with multiple risk factors for strokes and bleeds. A comprehensive approach to determining factors that increase bleed risk should be adopted. These factors include age (HAS-BLED, HEMORR $\mathrm{H}_{2} \mathrm{HAGES}, \mathrm{mOBRI}$, ATRIA); anemia (mOBRI, HEMORR ${ }_{2} \mathrm{HAGES}$, ATRIA); hepatic/ renal disease (HAS-BLED, HEMORR ${ }_{2}$ HAGES, ATRIA, mOBRI); concomitant medications/alcohol use, including NSAIDs, corticosteroids, and antiplatelet therapy (HAS$\mathrm{BLED}, \mathrm{HEMORR}{ }_{2} \mathrm{HAGES}$ ); bleed history/rebleeding risk
(HEMORR ${ }_{2}$ HAGES, HAS-BLED, ATRIA); and Gl bleeds $(\mathrm{mOBRI}){ }^{1,2}$ Additional risk factors for $\mathrm{Gl}$ bleeds include being a tobacco smoker and/or being infected with Helicobacter pylori. A prospective cohort study that analyzed data from questionnaires completed by 99,359 individuals from the Copenhagen General Population Study reported that the multivariable adjusted hazard ratio for current smokers versus never smokers was 2.20 (95\% $\mathrm{Cl}, 1.84-2.62)$ for $\mathrm{Gl}$ bleeds. ${ }^{25}$ Presence of $H$ pylori should be investigated, with a subsequent eradication regimen implemented, as patients with warfarin-associated upper Gl bleeds who were $H$ pylori-positive had lower HASBLED scores versus those who were negative. ${ }^{26}$

When bleed risk was lower than stroke risk (eg, HAS$\mathrm{BLED}<3, \mathrm{CHA}_{2} \mathrm{DS}_{2} \mathrm{VASc} \geq 1$ ), respondents appropriately initiated therapy with an OAC (predominantly apixaban); a small proportion also added gastroprotection. If the patient did not have any other Gl bleed risk factors (eg, a previous Gl bleed or on chronic antiplatelet or NSAID therapy), the choice of OACT depended on the attributes of each OAC and patient preference. ${ }^{27}$ Selection of warfarin was appropriate if cost, formulary restrictions, and availability of an inexpensive reversal agent were important concerns to patients and/or their health care providers. Rivaroxaban was selected because of its once-daily dosing and low risk for Gl bleeding.

The recently published ARISTOPHANES study provides evidence that apixaban is an appropriate choice in patients with a HAS-BLED score $<3$. In this retrospective observational study, more than $70 \%$ of patients received standard doses of DOACs (apixaban 5 mg, dabigatran $150 \mathrm{mg}$, or 


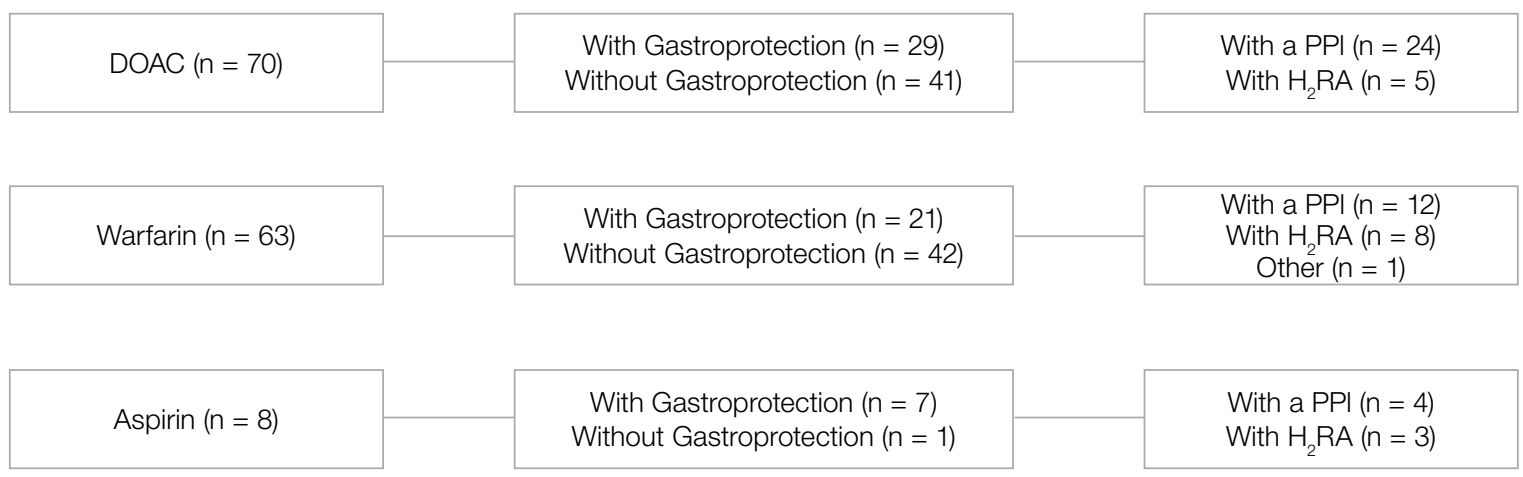

Figure 3. Pharmacists' treatment preferences if bleed risk is equal to stroke risk ( $n=141)$.

Data of 24 pharmacists are not included in figure, as they chose "other" treatment. DOAC, direct-acting oral anticoagulant (apixaban [n = 56]; rivaroxaban $[n=11]$; other [ $n=3]$; $P P I$, proton pump inhibitor; $H_{2} R A$, histamine-2 receptor antagonist.

rivaroxaban $20 \mathrm{mg}$ ) and about $20 \%$ had a bleeding history, about $30 \%$ were on PPls, less than $25 \%$ were on NSAIDs, and about $40 \%$ had a HAS-BLED score $<3$. The study found that apixaban was more effective (reduced rates of ischemic or hemorrhagic strokes/systemic embolism) and safer (reduced rates of major Gl bleed or intracranial bleed) than warfarin. ${ }^{28}$ Dabigatran and rivaroxaban were also more effective than warfarin for stroke prevention and had a lower risk for major intracranial bleed risk; while the risk of major Gl bleed was similar between dabigatran and warfarin, major Gl bleed risk was higher for rivaroxaban. When compared with each other, the 3 DOACs were effective at stroke prevention, with apixaban more effective than dabigatran and rivaroxaban; similar efficacy was noted for dabigatran versus rivaroxaban. Apixaban was associated with fewer $\mathrm{Gl}$ bleeds versus dabigatran and rivaroxaban, but with similar intracranial bleed risks; dabigatran was associated with fewer Gl bleeds but similar intracranial bleed risks versus rivaroxaban. ${ }^{28}$ Efficacy and safety findings from a subgroup analysis based on HAS-BLED scores $<3$ and $\geq 3$ were generally consistent with the main results.

When bleed risk was equal to stroke risk, the difficulty was determining how OACT in a patient at high stroke risk $\left(\mathrm{CHA}_{2} \mathrm{DS}_{2}\right.$ VASc score $\left.\geq 2\right)$ and high bleed risk (HAS-BLED score $\geq 3$ ) should be managed. Eight respondents chose aspirin and added gastroprotection with either a PPI or $\mathrm{H}_{2} \mathrm{RA}$; however, currently, aspirin is not recommended as the sole antithrombotic for patients with NVAF. ${ }^{23}$ With the OACT, an interesting finding was that as the number of patients seen in 12 months increased, pharmacists were almost twice as likely to select a DOAC over warfarin. Moreover, pharmacists were judicious in their recommendation to add gastroprotection, and would consider doing so if there was a specific indication. At the time of our survey, several studies described DOAC-associated Gl bleeds, ${ }^{29-31}$ but data on the effectiveness of acid-suppressive therapy, specifically with PPIs, in the prevention of upper Gl bleeds were sparse. ${ }^{4,7,32}$ Respondents most likely were familiar with GI bleed risk factors and prevention strategies from various guidelines published between 2009 and 2010, which did not include DOACs. ${ }^{33-35}$

Another important finding was pharmacists' uncertainty as to the effectiveness of PPIs in preventing Gl bleeds in combination with DOACs. The data are conflicting. A meta-analysis of older studies (2007-2015) showed that PPIs (but not $\mathrm{H}_{2} \mathrm{RAs}$ ) reduced the risk of upper $\mathrm{Gl}$ bleeds in patients on warfarin but not for dabigatran. ${ }^{36}$ A retrospective cohort study of Medicare beneficiaries on OACTs (2011-2015) showed the adjusted incidence of hospitalization for upper Gl bleeds in patients on PPI co-therapy was significantly lower compared with patients not on PPI co-therapy (76 versus 115 per 10,000 person-years, respectively). ${ }^{8}$ Apixaban without PPI co-therapy was associated with the lowest risk of upper 
Gl bleed hospitalizations (73/10,000 person-years), and PPI co-therapy further reduced this risk $(49 / 10,000$ person-years). Warfarin without PPI co-therapy was associated with the next lowest risk (113/10,000 person-years), followed by dabigatran (120/10,000 person-years) and rivaroxaban (144/10,000 person-years). PPI co-therapy significantly reduced the risk of upper Gl bleed hospitalizations with all OACTs, but the incidence of upper Gl bleed hospitalizations with rivaroxaban was significantly greater than with the other OACTs. ${ }^{8}$ Therefore, if there are concerns about the safety of PPIs, ${ }^{37-39}$ or the patient is unable to tolerate a PPI, then apixaban may be the most appropriate DOAC for a patient with high bleed risk. Notably, a 2020 review of data from the PINNACLE registry (average age, $75-77$ years; 31\% on PPIs) found that the relative $\mathrm{Gl}$ bleed safety advantage of apixaban and dabigatran versus warfarin was attenuated in patients $\geq 75$ years. ${ }^{40}$ Last, since the risk for lower Gl bleeds is not reduced by PPls, ${ }^{41}$ consideration of their use should be accompanied by an assessment to detect bleeds (eg, low hemoglobin/hematocrit, presence of bright red blood, hematochezia/melena, fecal occult testing), with prompt management as necessary. ${ }^{5}$

\section{Limitations}

Limitations of our survey included an overall low response rate, which can generate a biased sample if respondents are systematically different from nonrespondents. In addition, to maintain simplicity and reduce respondents' time commitment, our survey did not include actual $\mathrm{CHA}_{2} \mathrm{DS}_{2}$ VASc stroke risk scores, HAS-BLED bleed risk scores, or specific Gl bleed risk factors when querying pharmacists about treatment options based on bleed risk. The addition of these variables would have improved the robustness of the data.

\section{Conclusion}

In addition to applying BRTs in the management of NVAF patients, pharmacists considered patient-specific variables, prescriber preferences, and evidence-based guidance when recommending OACT with or without gastroprotection. To avoid suboptimal patient management, busy pharmacists should be granted time to attend continuing education programs describing opti- mal OACT selection and formulation of individualized, evidence-based plans to address modifiable risk factors for bleeding, including the appropriate use of gastroprotection. Randomized, prospective, long-term studies stratified by HAS-BLED and $\mathrm{CHA}_{2} \mathrm{DS}_{2}$ VASc scores are needed to further clarify efficacy, safety, and cost-effectiveness of OACT, with and without PPIs, in patients who may be at risk for upper Gl bleeds.

Acknowledgments: The authors thank Robin J. Jacobs, PhD, MSW, MS, MPH, Patrick C. Hardigan, PhD, Steven Brettler, PharmD, MPH, Maria-Isabel A. Cabral, PharmD, and Reginald Gyapong, PharmD, for their participation in this project. The authors also sincerely thank Fabio Franco, BS Computer Science, who organized the database to enable efficient data management.

Corresponding author: Devada Singh-Franco, PharmD, CDE, Nova Southeastern University College of Pharmacy, 3200 S University Drive, Fort Lauderdale, FL 33328; singh@nova.edu

Disclosures: None.

Funding: The study was supported by Nova Southeastern University's Health Professions Division Internal Research Grant.

\section{doi:10.12788/jcom.0033}

\section{References}

1. Apostolakis S, Lane DA, Guo $Y$, et al. Performance of the HEMORR2HAGES, ATRIA, and HAS-BLED bleeding risk-prediction scores in patients with atrial fibrillation undergoing anticoagulation. $J$ Am Coll Cardiol. 2012;60:861-867.

2. Chang $G$, Xie $Q, M a ~ L$, et al. Accuracy of HAS-BLED and other bleeding risk assessment tools in predicting major bleeding events in atrial fibrillation: A network meta-analysis. J Thromb Haemost. 2020;18:791-801.

3. Ding WY, Harrison SL, Lane DA, Lip GYH. Considerations when choosing an appropriate bleeding risk assessment tool for patients with atrial fibrillation. J Thromb Haemost. 2020;18:788-790.

4. Lauffenburger JC, Rhoney DH, Farley JF, et al. Predictors of gastrointestinal bleeding among patients with atrial fibrillation after initiating dabigatran therapy. Pharmacotherapy. 2015;35:560-568.

5. Tomaselli GF, Mahaffey KW, Cuker A, et al. 2020 ACC Expert Consensus Decision Pathway on Management of Bleeding in Patients on Oral Anticoagulants: A Report of the American College of Cardiology Solution Set Oversight Committee. J Am Coll Cardiol. 2020;76:594-622.

6. Taha A, McCloskey C, Craigen T, Angerson W. Antiplatelet versus anticoagulant effects in non-variceal upper gastrointestinal bleeding. Gut. 2019;68(suppl 2):A152.

7. Chan EW, Lau WC, Leung WK, et al. Prevention of dabigatran-related gastrointestinal bleeding with gastroprotective agents: A population-based study. Gastroenterology. 2015;149:586-595.

8. Ray WA, Chung CP, Murray KT, et al. Association of oral anticoagulants and proton pump inhibitor cotherapy with hospitalization for upper gastrointestinal tract bleeding. JAMA. 2018;320: 2221-2230. 
9. Brunetti L, Lee S-M, Doherty N, et al. Impact of warfarin discharge education program on hospital readmission and treatment costs. Int J Clin Pharm. 2018;40:721-729.

10. Hasan SS, Kow CS, Curley LE, et al. Economic evaluation of prescribing conventional and newer oral anticoagulants in older adults. Expert Rev Pharmacoecon Outcomes Res. 2018;18:371-377.

11. Phelps E, Delate T, Witt DM, et al. Effect of increased time in the therapeutic range on atrial fibrillation outcomes within a centralized anticoagulation service. Thromb Res. 2018;163:54-59.

12. Ahuja T, Raco V, Papadopoulos J, Green D. Antithrombotic stewardship: Assessing use of computerized clinical decision support tools to enhance safe prescribing of direct oral anticoagulants in hospitalized patients. J Patient Saf. 2018 Sep 25. [Epub ahead of print]

13. Leef GC, Perino AC, Askari M, et al. Appropriateness of direct oral anticoagulant dosing in patients with atrial fibrillation: Insights from the Veterans Health Administration. J Pharm Pract. 2020;33:647-653.

14. Papastergiou J, Kheir N, Ladova K, et al. Pharmacists' confidence when providing pharmaceutical care on anticoagulants, a multinational survey. Int J Clin Pharm. 2017;39:1282-1290.

15. Perlman A, Horwitz E, Hirsh-Raccah B, et al. Clinical pharmacist led hospital-wide direct oral anticoagulant stewardship program. Isr J Health Policy Res. 2019;8:19.

16. Uppuluri EM, McComb MN, Shapiro NL. Implementation of a direct oral anticoagulation screening service at a large academic medical center provided by a pharmacist-managed antithrombosis clinic as a method to expand antithrombotic stewardship efforts. J Pharm Pract. 2020;33:271-275.

17. Manzoor BS, Cheng W-H, Lee JC, et al. Quality of pharmacistmanaged anticoagulation therapy in long-term ambulatory settings: A systematic review. Ann Pharmacother. 2017;51:1122-1137.

18. Harris PA, Taylor R, Thielke R, et al. Research Electronic Data Capture (REDCap)-A metadata-driven methodology and workflow process for providing translational research informatics support. $J$ Biomed Inform. 2009;42:377-381.

19. Brooks M. AF management: Are clinicians in agreement? Medscape. May 30, 2019. Accessed December 29, 2020. https:// www.medscape.com/viewarticle/913386

20. Amroze A, Mazor K, Crawford S, et al. Survey of confidence in use of stroke and bleeding risk calculators, knowledge of anticoagulants, and comfort with prescription of anticoagulation in challenging scenarios: SUPPORT-AF II study. J Thromb Thrombolysis. 2019;48:629-637.

21. Wang $Y$, Bajorek B. Decision-making around antithrombotics for stroke prevention in atrial fibrillation: the health professionals' views. Int J Clin Pharm. 2016;38:985-995.

22. January CT, Wann LS, Alpert JS, et al. 2014 AHAVACC/HRS guideline for the management of patients with atrial fibrillation: a report of the American College of Cardiology/American Heart Association Task Force on Practice Guidelines and the Heart Rhythm Society. Circulation. 2014;130:e199-e267.

23. January CT, Wann LS, Calkins H, et al. 2019 AHAVACC/HRS Focused Update of the 2014 AHA/ACC/HRS Guideline for the Management of Patients With Atrial Fibrillation: A Report of the American College of Cardiology/American Heart Association Task Force on Clinical Practice Guidelines and the Heart Rhythm Society. J Am Coll Cardiol. 2019;74:104-132.

24. Anghel L, Sascu R, Trifan A, et al. Non-vitamin K antagonist oral anticoagulants and the gastrointestinal bleeding risk in real-world studies. J Clin Med. 2020;9:1398.
25. Langsted A, Nordestgaard BG. Smoking is associated with increased risk of major bleeding: a prospective cohort study. Thromb Haemost. 2019;119:39-47.

26. Faye AS, Hung KW, Cheng K, et al. HAS-BLED scores underestimate gastrointestinal bleeding risk among those with $\mathrm{H}$. pylori. $A m$ J Gastroenterol. 2019;114:S364.

27. Fawzy AM, Yang W-Y, Lip GY. Safety of direct oral anticoagulants in real-world clinical practice: translating the trials to everyday clinical management. Expert Opin Drug Saf. 2019;18:187-209.

28. Lip GYH, Keshishian A, Li X, et al. Effectiveness and safety of oral anticoagulants among nonvalvular atrial fibrillation patients. Stroke. 2018;49:2933-2944.

29. Abraham NS, Singh S, Alexander GC, et al. Comparative risk of gastrointestinal bleeding with dabigatran, rivaroxaban, and warfarin: population based cohort study. BMJ. 2015;350:h1857.

30. Holster IL, Valkhoff VE, Kuipers EJ, Tjwa E. New oral anticoagulants increase risk for gastrointestinal bleeding: a systematic review and meta-analysis. Gastroenterology. 2013;145:105-112.

31. Sherwood MW, Nessel CC, Hellkamp AS, et al. Gastrointestinal bleeding in patients with atrial fibrillation treated with rivaroxaban or warfarin: ROCKET AF Trial. J Am Coll Cardiol. 2015;66: 2271-2281.

32. Di Minno A, Spadarella G, Spadarella E, et al. Gastrointestinal bleeding in patients receiving oral anticoagulation: Current treatment and pharmacological perspectives. Thromb Res. 2015;136:1074-1081.

33. Abraham NS, Hlatky MA, Antman EM, et al. ACCF/ACG/AHA 2010 Expert Consensus Document on the Concomitant Use of Proton Pump Inhibitors and Thienopyridines: A Focused Update of the ACCF/ACG/AHA 2008 Expert Consensus Document on Reducing the Gastrointestinal Risks of Antiplatelet Therapy and NSAID Use. Circulation. 2010;122:2619-2633.

34. Bhatt DL, Scheiman J, Abraham NS, et al. ACCF/ACG/AHA 2008 expert consensus document on reducing the gastrointestinal risks of antiplatelet therapy and NSAID use: a report of the American College of Cardiology Foundation Task Force on Clinical Expert Consensus Documents. J Am Coll Cardiol. 2008;52:1502-1517.

35. Lanza FL, Chan FK, Quigley EM. Guidelines for prevention of NSAID-related ulcer complications. Am J Gastroenterol. 2009; 104:728-738.

36. Bang CS, Joo MK, Kim BW, et al. The role of acid suppressants in the prevention of anticoagulant-related gastrointestinal bleeding: a systematic review and meta-analysis. Gut Liver. 2020;14: 57-66.

37. Farrell B, Pottie K, Thompson W, et al. Deprescribing proton pump inhibitors: Evidence-based clinical practice guideline. Can Fam Physician. 2017;63:354-364.

38. Fossmark R, Martinsen TC, Waldum HL. Adverse effects of proton pump inhibitors-evidence and plausibility. Int $J \mathrm{Mol}$ Sci. 2019;20:5203.

39. Haastrup PF, Thompson W, Sondergaard J, Jarbol DE. Side effects of long-term proton pump inhibitor use: A review. Basic Clin Pharmacol Toxicol. 2018;123:114-121.

40. Wong JM, Maddox TM, Kennedy K, Shaw RE. Comparing major bleeding risk in outpatients with atrial fibrillation or flutter by oral anticoagulant type (from the National Cardiovascular Disease Registry's Practice Innovation and Clinical Excellence Registry). Am J Cardiol. 2020;125:1500-1507.

41. Nagata N, Niikura R, Aoki T, et al. Effect of proton-pump inhibitors on the risk of lower gastrointestinal bleeding associated with NSAIDs, aspirin, clopidogrel, and warfarin. J Gastroenterol. 2015;50:1079-1086. 